\title{
Brain Imaging in Cases with Positive Serology for Dengue with Neurologic Symptoms: A Clinicoradiologic Correlation
}

(D) H.A. Vanjare, (DP. Mannam, (D)A.K. Mishra, (D) Karuppusami, (DR.A.B. Carey, (D)A.M. Abraham, DW. Rose, (D). lyyadurai, and (1)S. Mani

\begin{abstract}
BACKGROUND AND PURPOSE: Dengue is a common arboviral disease, which uncommonly involves the brain. There has been a recent surge in dengue cases and dengue-related deaths in tropical countries. The aim of this study was to describe brain imaging findings in patients with dengue infection having neurologic symptoms.
\end{abstract}

MATERIALS AND METHODS: Thirty-five patients with positive serology for dengue with CNS symptoms undergoing imaging of the brain were included in the study. Clinical, laboratory, and imaging parameters were assessed and correlated to poor outcome.

RESULTS: A Glasgow Coma Scale score of $\leq 12$ at presentation, clinical classification of severe-type dengue, and the presence of acute renal failure were associated with poor outcome. Imaging parameters associated with poor outcome were involvement of the thalami and cerebellar peduncles and the presence of diffusion restriction and hemorrhagic foci in the brain parenchyma.

CONCLUSIONS: Although not specific, dengue infection has imaging findings that can be used to narrow down the differential list and help in prognostication.

ABBREVIATIONS: GCS = Glasgow Coma Scale; PRES = posterior reversible encephalopathy syndrome

D engue is an arboviral disease found in the tropics; it is the second most common mosquito-borne disease. It is transmitted by the bite of Aedes mosquitoes, which are primarily found in the tropics where the mosquitoes flourish in stagnant water. ${ }^{1}$ The virus has 4 different serotypes. Infection with 1 serotype (primary infection) does not provide life-long immunity from infection through other serotypes. Other serotypes can subsequently infect the same person and cause secondary infection. There has been a recent surge in dengue cases, and it has been reported from $>100$ countries. $^{2}$ Fifty million cases are estimated to occur globally per year. ${ }^{1}$ Following a bite from an infected mosquito, symptoms can occur after 4-10 days of incubation with abrupt onset of fever called the "febrile phase." Patients can have nonspecific symptoms such as myalgia, headache, skin erythema, and vomiting. Petechiae and mucosal hemorrhage can occur during this

Received June 23, 2017; accepted after revision November 29.

From the Departments of Radiology (H.A.V., P.M., S.M.), Medicine (A.K.M., R.A.B.C., R.I.), Biostatistics (R.K.), Virology (A.M.A.), and Pediatrics (W.R.), Christian Medical College and Hospital, Tamil Nadu, India.

Please address correspondence to Sunithi Mani, MD, Department of Radiology, Christian Medical College and Hospital, Ida Scudder Rd, Vellore-632004, India; e-mail: sunithi.mani@cmcvellore.ac.in

5 Indicates article with supplemental on-line photos.

三 Indicates article with supplemental on-line table.

http://dx.doi.org/10.3174/ajnr.A5544 phase. This is followed by the "critical phase," in which capillary permeability increases, leading to hemoconcentration with associated thrombocytopenia. Significant plasma leak can occur during this phase with associated multiorgan dysfunction. Most patients gradually improve during the "recovery phase," in which extravascular fluid is reabsorbed with improvement in platelet count. Neurologic involvement of dengue infection is uncommon and can be seen in $4 \%-5 \%$ of those infected, and includes encephalopathy, acute disseminated encephalomyelitis, branchial neuritis, myelitis, hypokalemic paralysis, opsoclonus myoclonus, and Guillain-Barré syndrome. ${ }^{3-5}$ Dengue is known to cause encephalitis (for which the hallmark is altered sensorium and seizures); however, multiple metabolic derangements, which usually occur with dengue infection, can also lead to a similar clinical presentation (encephalopathy). Therefore, clinical distinction between dengue encephalitis and encephalopathy is often not possible. Because of rare CNS involvement in dengue, primary care physicians may be unfamiliar with its neurologic manifestations; in addition, there are insufficient data with respect to brain imaging findings in dengue infection, with most of the data published as case reports or short case series.

These cases were included from a tertiary care nongovernment medical college hospital in South India. Cases presenting to this institution are often referrals from surrounding primary and sec- 
ondary care medical facilities where management of complicated cases is difficult. Patients seen in this hospital are from poor socioeconomic backgrounds. This causes constraint on the investigation that a physician can request because most patients are self-paid.

\section{MATERIALS AND METHODS}

A single-center retrospective chart review was performed with the cases identified using the case data base of the radiology department. Thirty-five patients with acute dengue infection with symptoms suggestive of CNS involvement undergoing imaging of the brain were included in the study (duration, 2006 to 2017). Both CT and MR imaging scans were included. There were an additional 4 patients for whom there was no imaging available during the acute phase of the illness; however, follow-up imaging was available. These cases were not included in the data analysis but are described briefly.

Clinical parameters assessed were the following: age and sex of the patients; type of dengue infection (primary versus secondary); Glasgow Coma Scale (GCS) score at presentation; the presence of seizures, vomiting, and hemorrhagic manifestations (such as mucosal hemorrhage, hematemesis, hematochezia or excessive menstrual flow); the requirement of intubation and inotropic support; the presence of hospital-acquired pneumonia; the World Health Organization (2009) classification of dengue infection; the lowest documented platelet count; liver dysfunction (hyperbilirubinemia and transaminitis); deranged prothrombin time and activated partial thromboplastin time; acute renal failure; sepsis (positive blood culture); urinary tract infection; and CSF analysis if performed.

Indications for imaging were documented. MR imaging was performed on 1.5T (Magnetom Avanto; Siemens, Erlangen, Germany) or 3T (Intera Achieva; Philips Healthcare, Best, the Netherlands) scanners while CT was performed on 6-slice (Brilliance 6; Philips Healthcare) or 64-slice (Discovery 750 HD; GE Healthcare, Milwaukee, Wisconsin) scanners. Scans were reviewed by 2 radiologists with 2 and 4 years of experience in neuroradiology working simultaneously on a single workstation. Any disagreement was resolved by consensus. In the absence of consensus, images were reviewed by a neuroradiologist with 10 years' experience whose decision was considered final. Studies were assessed for the following: supratentorial white matter changes (periventricular, deep, and subcortical white matter; assessed for symmetricity), infratentorial white matter (brain stem, middle cerebellar peduncles, and cerebellar white matter; assessed for symmetricity), involvement of gray matter (cortical gray matter, basal ganglia, and thalami), the presence of diffusion restriction (cerebral white matter, deep gray matter, brain stem, and cerebellum), and the presence of hemorrhage (micro- [small foci of hemorrhage on CT or small foci of blooming of susceptibilityweighted images on MR imaging] and macrohemorrhage).

Final outcome was assessed and divided into good outcome for patients discharged in stable condition with no neurologic deficit versus poor outcome for death or the presence of neurologic deficits at discharge.

\section{Statistical Analysis}

All the cases were divided into 2 categories of poor-versus-good outcome, and each of the clinical, laboratory, and imaging parameters was assessed individually using $\chi^{2}$ and Fisher exact tests to look for any significant association with poor outcome (Online Table). All analyses were performed using the Statistical Package for Social Sciences software, Version 21.0 (IBM, Armonk, New York). A GCS score of $\leq 12$ at presentation, World Health Organization (2009) classification of dengue as severe type, and the presence of acute renal failure had significant association with poor outcome $(P=.04, .02$, and .03 respectively). Imaging parameters that had significant association with poor outcome were thalamic involvement $(P=.005)$, cerebellar peduncle involvement $(P=.01)$, the presence of diffusion restriction $(P=.01)$, and the presence of hemorrhage (both micro- and macrohemorrhage, $P=.003$ ).

\section{RESULTS}

Fourteen $(40 \%)$ patients were children (15 years of age or younger; mean, 6.8 years) and 21 (60\%) were adults (mean, 33.2 years). Clinical and laboratory parameters were assessed as described in the On-line Table. CSF analysis was performed in 6 cases. Three patients had normal CSF analysis and 2 had a marginal increase in white blood cells with normal proteins, and 1 patient had normal cells but marginally elevated proteins. CSF was not tested for the dengue antigen.

\section{Type of Dengue Infection}

There were 8 cases with primary infection (positive for serum immunoglobulin $\mathrm{M}$ and negative for immunoglobulin $\mathrm{G}$ for dengue. In 1 case, both immunoglobulin $\mathrm{M}$ and immunoglobulin $\mathrm{G}$ were negative; however, the nonstructural protein 1 antigen was positive). Twenty-seven cases had secondary infection (serum immunoglobulin G-positive with or without positive serum immunoglobulin $\mathrm{M}$ ). Of the 22 patients in whom the serum nonstructural protein 1 antigen test was available, it was positive in 9 .

\section{World Health Organization (2009) Classification of Dengue}

One case was classified as dengue without warning signs, 3 cases were classified as dengue with warning signs, and 31 cases were classified as severe dengue.

\section{Indications for Imaging}

One case had an acute drop in sensorium, 18 cases had altered sensorium, 8 cases had altered sensorium with seizures, 5 cases had acute onset headache, and intracranial hemorrhage was suspected in 3 cases.

\section{Imaging Findings}

Nine cases had both MR imaging and CT scans, 7 cases had only MR imaging, and 19 cases had only CT scans. Fifteen (42.9\%) cases did not have any significant abnormality on imaging (Online Figures 1-4). 
Table 1: White and gray matter abnormalities in different brain regions on both CT and MRI scans ( 35 cases)

\begin{tabular}{lrc}
\hline \multicolumn{1}{c}{ Patterns of Involvement } & No. & $\%$ \\
\hline White matter & 14 & $40.0 \%$ \\
Cerebral white matter & 3 & \\
Symmetric & 10 & \\
Asymmetric & 4 & \\
Periventricular white matter & 9 & \\
Deep white matter & 11 & \\
Subcortical U-fibers & 11 & $31.4 \%$ \\
Cerebellar white matter & 3 & \\
$\quad$ Peripheral & 5 & \\
Both central and peripheral & 4 & \\
Symmetric & 7 & \\
Asymmetric & 8 & $22.9 \%$ \\
Middle cerebellar peduncles & 2 & $5.7 \%$ \\
Basal ganglia & 9 & $25.7 \%$ \\
Thalamus & 6 & \\
Symmetric & 3 & \\
Asymmetric & 3 & $8.6 \%$ \\
Substantia nigra & 1 & \\
Asymmetric & 2 & \\
Symmetric & 10 & $28.6 \%$ \\
Brain stem &
\end{tabular}

\section{Involvement of White and Gray Matter on T1-Weighted/T2-Weighted Sequences and CT}

Of the 9 patients who had both MR imaging and CT scans performed in a short interval (within 1 week), there was concordance of the imaging findings in 8 cases. One case demonstrated involvement of the substantia nigra on MR imaging, which could not be appreciated on CT.

Dengue infection is known to involve cortical gray matter, subcortical and deep white matter, the basal ganglia, thalami, brain stem, and cerebellum. ${ }^{6-8}$ In 1 series, all $(n=8)$ cases had involvement of the cerebellum. ${ }^{9}$ In our series (Table 1), posterior structures such as the brain stem and cerebellum were more commonly involved than anterior structures such as the basal ganglia. A clear gradient was found in which there was progressive involvement of basal ganglia, thalami, brain stem, and cerebellum in an increasing order. While involvement of thalami is commonly seen in patients with Japanese encephalitis and is considered quite specific for this disease, ${ }^{10,11}$ there were 9 cases $(25.7 \%)$ with involvement of thalami in the current series with 1 case showing pure thalamic involvement. With respect to involvement of the cerebellum (31.4\% of the cases), peripherally located structures were involved more commonly, either in isolation or in combination with central structures rather than central structures alone. Involvement of cerebral white matter was also found in $40 \%$ of the cases. Again, a superficial-to-deep gradient was observed in which subcortical white matter was involved more commonly than deep white matter. Cerebral white matter involvement was found to be predominantly asymmetric.

\section{Involvement of the Substantia Nigra}

Viral encephalitis is very rarely known to involve the substantia nigra with Parkinsonism-like late clinical presentation. ${ }^{12}$ Encephalitis related to West Nile virus, Murray Valley encephalitis, Epstein-Barr virus, and, more commonly, Japanese encephalitis is known to involve the substantia nigra as an exception rather than a rule. ${ }^{13-16}$ In the current series, 3 cases were found to have substantia nigra involvement.
Table 2: Diffusion restriction in cases with MRI scans (16 cases)

\begin{tabular}{lcc}
\hline \multicolumn{1}{c}{ Patterns } & No. & $\%$ \\
\hline Diffusion restriction present & 11 & $68.8 \%$ \\
Cerebral white matter involvement & 7 & $43.8 \%$ \\
Deep gray matter involvement & 8 & $50.0 \%$ \\
Brain stem involvement & 6 & $37.5 \%$ \\
Cerebellar involvement & 6 & $37.5 \%$ \\
\hline
\end{tabular}

\section{Diffusion Restriction}

Eleven $(68.8 \%)$ of the 16 patients who underwent MR imaging had focal areas of diffusion restriction. Areas of diffusion restriction were found to involve both gray and white matter in both supra- and infratentorial regions (Table 2). In 1 case, there was diffusion restriction involving the head of the bilateral hippocampi, while 1 case had focal splenial hyperintensity with associated diffusion restriction. In patients with thalamic involvement, thalami were symmetrically swollen, with T2-weighted hyperintensity and central diffusion restriction.

Diffusion restriction is known to be involved in cases with viral encephalitis with studies describing areas of diffusion restriction in cytomegalovirus, herpes simplex, influenza, enterovirus 71related encephalitis, and John Cunningham virus-related progressive multifocal leukoencephalopathy among others. ${ }^{17-21}$ There are few data with respect to diffusion restriction with dengue infection, with case reports describing the presence of diffusion restriction in the thalami, splenium of the corpus callosum, ${ }^{22}$ and pons. $^{23}$

Acute arterial territory strokes have been described as a rare complication in dengue infection. ${ }^{24}$ In the current series, there was 1 case for which only follow-up imaging was available, showing chronic lacunar infarcts in the bilateral basal ganglia region.

\section{Microhemorrhages}

Of the 10 cases, 8 had MR imaging scans in which microhemorrhages were seen as small foci of blooming on susceptibilityweighted images, while they were seen as high-density foci on CT. Microhemorrhages were seen involving the thalami in 5 cases, the pons in 4 cases, and the cerebral and cerebellar white matter in 2 cases each. Six patients who had microhemorrhages on MR imaging also underwent CT at close intervals (within 1 week). Microhemorrhages could not be appreciated on these CT scans.

\section{Macrohemorrhages}

Macrohemorrhage was found in 3 cases on CT. In the first case, there was acute intraventricular hemorrhage with a normal platelet count at the time of imaging. In the second case, there was acute left cerebellar hematoma with posteriorly located subdural hemorrhage along the posterior fossa and extension along bilateral tentorial leaves. In the third case, there was acute right cerebellar hematoma with associated right parietal subdural hemorrhage extending along the tentorium. Both cases with acute cerebellar hematoma had low platelet counts (5000 and 29,000/ $\mathrm{mm}^{3}$ ) at the time of imaging.

Dengue encephalitis with associated acute hemorrhage is termed "dengue hemorrhagic encephalitis." These hemorrhages can be tiny or large and intra- or extra-axial. Hemorrhages are known to rarely occur in the basal ganglia, pituitary gland, pons, cerebellum, and subdural or intraventricular locations or may 
present as multiple small intraparenchymal hemorrhagic foci. $^{25-28}$ In the current series, there were 13 (37.1\%) cases with hemorrhages, the pattern suggestive of posterior predominance (involvement of thalami, pons, and cerebellar white matter).

\section{Posterior Reversible Encephalopathy Syndrome}

Two patients had white matter edema involving the bilateral occipital and parietal regions in a fairly symmetric distribution with associated increases in blood pressure during their hospital stay suggestive of posterior reversible encephalopathy syndrome (PRES). The presence of PRES in a case with dengue infection has been described previously. ${ }^{29}$

\section{Outcome following an Acute Episode}

Poor outcome was seen in 21 patients There were 9 deaths in the hospital, 2 patients were discharged at their request in a critical state for end-of-life care at home, 4 patients had decreased sensorium at discharge ( 1 of whom had gaze palsy), 6 patients were alert at discharge with varying deficits (1 right hemiparesis, 1 ataxia, 2 swallowing dysfunctions requiring nasogastric feeding, 1 truncal weakness, and 1 patient with psychiatric symptoms in the form of anger outbursts, irritability, memory deficits, and delusions of infidelity causing loss of employment on follow-up). Of the 15 cases with normal findings on imaging studies, 9 cases had good outcome and 6 cases had poor outcome.

\section{Cause of Death}

No postmortem examination was performed. All the cases had multiorgan dysfunction with 3 cases documented with disseminated intravascular coagulation.

\section{Follow-Up Imaging}

Follow-up imaging was available in 6 cases at mean interval of 4.7 months. On the initial scans, swelling and T2-weighted hyperintensity were seen in the pons in 5 cases; the cerebellum in 4 cases; the thalami in 3 cases; and periventricular, deep, and subcortical U-fibers in 2 cases each with cortical gray matter involvement in 1 case. On follow-up imaging, the swelling in these regions had resolved with volume loss and residual gliotic changes. In 1 case with symmetric involvement of the substantia nigra and another case with cortical gray matter involvement, no residual gliotic changes could be appreciated.

Additionally, in 4 cases (not included in data analysis) only follow-up imaging was available. The first case was a 2 -year-old boy admitted with severe dengue infection. At discharge, he had regression of developmental milestones in the form of partial loss of head control and required support to sit. MRI at 1 month after the acute episode demonstrated chronic lacunar infarcts in the bilateral caudate nuclei, left lentiform nucleus, and anterior limb of the left internal capsule. The second case was a 4-year-old boy admitted with severe dengue infection. At discharge, he had swallowing dysfunction and required feeding via a nasogastric tube. One month after the acute episode, MRI scan showed normal imaging findings. The third and fourth patients, 18 and 22 years of age respectively, were both admitted with severe dengue but were discharged in stable condition with no neurologic deficits. MR imaging was performed at 2 months and 2 weeks, respectively, for both the patients after the acute episode. Findings of these scans were normal.

\section{DISCUSSION}

Dengue is an RNA virus and has come into prominence due to the high social and economic cost associated with the infections it causes, especially because it involves underdeveloped parts of the world. $^{30}$ Although dengue virus causes multisystem disease, which can lead to multiorgan dysfunction, it rarely has neurologic manifestation. Neurologic involvement is of concern because of long-term sequelae that can be seen in such cases.

Following a bite by an infected mosquito, the dengue virus can replicate in lymph nodes, muscles, and fibroblasts. Macrophages can also be infected. The virus can access the brain parenchyma either by disruption of the blood-brain barrier or secondary to movement of the infected macrophage from peripheral blood into the CSF or brain parenchyma. ${ }^{31}$ Postmortem studies have isolated the dengue antigen from the brain tissue. ${ }^{32}$ The actual mechanism of brain injury is still debated and can be attributed to direct viral invasion, metabolic imbalance, hemorrhagic diathesis, or postinfection autoimmunity. ${ }^{31}$ Current research is limited by the lack of a good animal model. Therefore, in vivo imaging studies can help further our understanding.

A large proportion of cases in the current series did not have any significant abnormal findings on their cranial scans ( 15 cases, 42.9\%). The apparent lack of correlation between CNS symptoms and normal imaging findings could be related to metabolic derangement that was present in these cases.

Among the cases with abnormal brain imaging findings, this study demonstrates involvement of important brain structures such as the brain stem, cerebellum, thalami, and cerebral white matter, which could explain the significant morbidity (12 cases, 34.3\%) and mortality ( 9 cases, $25.7 \%$ ). The imaging findings in this series can also be seen in other Flavivirus infections such as Japanese encephalitis, West Nile virus encephalitis, and Murray valley virus encephalitis, suggestive of similar pathophysiology. ${ }^{10-14}$

Therefore, although accurate identification of dengue-related brain changes cannot be made purely on the basis of imaging findings, the differential diagnosis can be significantly reduced. With the additional input of typical clinical presentation, thrombocytopenia, and local prevalence of the disease, one cannot only suggest the possibility of dengue infection but can also help in prognosis based on involvement of the thalami and cerebellar peduncles, and the presence of diffusion restriction and intraparenchymal hemorrhage.

\section{Limitations}

This was a retrospective study with selection bias because only very sick individuals with CNS symptoms underwent imaging of the brain. Therefore, the results can be extrapolated to only a small similar subgroup of patients.

\section{CONCLUSIONS}

Although imaging findings are not unique, in the presence of CNS symptoms, dengue infection has radiological findings that can be used to narrow down the differential diagnosis list and help in prognostication. Development of an animal model will help to 
better understand the pathophysiology of CNS involvement in dengue infection. This could also help in the development of neuroprotective measures.

\section{REFERENCES}

1. Dengue: Guidelines for Diagnosis, Treatment, Prevention and Control. Geneva: World Health Organization; 2009 (WHO Guidelines Approved by the Guidelines Review Committee). http://www.ncbi. nlm.nih.gov/books/NBK143157/. Accessed April 18, 2017

2. WHO. Epidemiology. Dengue control. http://www.who.int/ denguecontrol/epidemiology/en/. Accessed November 13, 2017

3. Solomon T, Dung NM, Vaughn DW, et al. Neurological manifestations of dengue infection. Lancet 2000;355:1053-59 CrossRef

4. Pancharoen C, Thisyakorn U. Neurological manifestations in dengue patients. Southeast Asian J Trop Med Public Health 2001;32: 341-45 Medline

5. Verma R, Sharma P, Garg RK, et al. Neurological complications of dengue fever: experience from a tertiary center of north India. Ann Indian Acad Neurol 2011;14:272-78 CrossRef Medline

6. Bhoi SK, Naik S, Kumar S, et al. Cranial imaging findings in dengue virus infection. J Neurol Sci 2014;342:36-41 CrossRef Medline

7. Kamble R, Peruvamba JN, Kovoor J, et al. Bilateral thalamic involvement in dengue infection. Neurol India 2007;55:418-19 CrossRef Medline

8. Wasay M, Channa R, Jumani M, et al. Encephalitis and myelitis associated with dengue viral infection clinical and neuroimaging features. Clin Neurol Neurosurg 2008;110:635-40 CrossRef Medline

9. Hegde V, Aziz Z, Kumar S, et al. Dengue encephalitis with predominant cerebellar involvement: report of eight cases with MR and CT imaging features. Eur Radiol 2015;25:719-25 CrossRef Medline

10. Basumatary LJ, Raja D, Bhuyan D, et al. Clinical and radiological spectrum of Japanese encephalitis. J Neurol Sci 2013;325:15-21 CrossRef Medline

11. Misra UK, Kalita J. Overview: Japanese encephalitis. Prog Neurobiol 2010;91:108-20 CrossRef Medline

12. Rebai I, Ben Rhouma H, Kraoua I, et al. Postencephalitic parkinsonism and selective involvement of substantia nigra in childhood. Brain Dev 2015;37:153-57 CrossRef Medline

13. Bosanko CM, Gilroy J, Wang AM, et al. West Nile virus encephalitis involving the substantia nigra: neuroimaging and pathologic findings with literature review. Arch Neurol 2003;60:1448-52 CrossRef Medline

14. Einsiedel L, Kat E, Ravindran J, et al. MR findings in Murray Valley encephalitis. AJNR Am J Neuroradiol 2003;24:1379-82 Medline

15. Çelik T, Çelik Ü, Tolunay O, et al. Epstein-Barr virus encephalitis with substantia nigra involvement. J Pediatr Neurosci 2015;10: 401-03 CrossRef Medline

16. Ogata A, Tashiro K, Pradhan S. Parkinsonism due to predominant involvement of substantia nigra in Japanese encephalitis. Neurology 2000;55:602 Medline

17. Renard T, Daumas-Duport B, Auffray-Calvier E, et al. Cytomegalovirus encephalitis: undescribed diffusion-weighted imaging char- acteristics - original aspects of cases extracted from a retrospective study, and from literature review. J Neuroradiol 2016;43:371-77 CrossRef Medline

18. Sener RN. Herpes simplex encephalitis: diffusion MR imaging findings. Comput Med Imaging Graph 2001;25:391-97 CrossRef Medline

19. Bulakbasi N, Kocaoglu M, Tayfun C, et al. Transient splenial lesion of the corpus callosum in clinically mild influenza-associated encephalitis/encephalopathy. AJNR Am J Neuroradiol 2006;27: 1983-86 Medline

20. Lian Z, Huang B, He S, et al. Diffusion-weighted imaging in the diagnosis of enterovirus 71 encephalitis. Acta Radiol 2012;53: 208-13 Medline

21. Barata Tavares J, Geraldo AF, Neto L, et al. Diffusion-weighted MR imaging characterization of progressive multifocal leucoencefalopathy (PML) lesions [in Portuguese]. Acta Med Port 2012;25(Suppl 1):34-37 Medline

22. Pal S, Sen K, Biswas NM, et al. Clinico-radiological profile and outcome of dengue patients with central nervous system manifestations: a case series in an Eastern India tertiary care hospital. J Neurosci Rural Pract 2016;7:114-24 CrossRef Medline

23. Mehta A, Mahale RR, Javali M, et al. Diffusion restriction in pons resembling "reverse moustache" in dengue encephalitis. Neurol India 2014;62:683-84 CrossRef Medline

24. Mathew S, Pandian JD. Stroke in patients with dengue. J Stroke Cerebrovasc Dis 2010;19:253-56 CrossRef Medline

25. Kumar R, Prakash O, Sharma BS. Intracranial hemorrhage in dengue fever: management and outcome: a series of 5 cases and review of literature. Surg Neurol 2009;72:429-33; discussion 433 CrossRef Medline

26. Kumar V, Kataria R, Mehta VS. Dengue hemorrhagic fever: a rare cause of pituitary tumor hemorrhage and reversible vision loss. Indian J Ophthalmol 2011;59:311-12 CrossRef Medline

27. Kumar R, Prakash O, Sharma BS. Dengue hemorrhagic fever: a rare presentation as atypical acute subdural hematoma. Pediatr Neurosurg 2008;44:490-92 CrossRef Medline

28. de Souza LJ, Martins AL, Paravidini PC, et al. Hemorrhagic encephalopathy in dengue shock syndrome: a case report. Braz J Infect Dis 2005;9:257-61 Medline

29. Sohoni CA. Bilateral symmetrical parieto occipital involvement in dengue infection. Ann Indian Acad Neurol 2015;18:358-59 CrossRef Medline

30. Dengue: Guidelines for Diagnosis, Treatment, Prevention and Control: New Edition. Epidemiology, Burden of Disease and Transmission. https:/www.ncbi.nlm.nih.gov/books/NBK143159/. Accessed November 14, 2017

31. Puccioni-Sohler M, Rosadas C. Advances and new insights in the neuropathogenesis of dengue infection. Arq Neuropsiquiatr 2015;73: 698-703 CrossRef Medline

32. Bhoopat L, Bhamarapravati N, Attasiri C, et al. Immunohistochemical characterization of a new monoclonal antibody reactive with dengue virus-infected cells in frozen tissue using immunoperoxidase technique. Asian Pac J Allergy Immunol 1996; 14: 107-13 Medline 